\title{
Interdisciplinary management of hip fracture
}

\author{
Authors: Cameron Swift, ${ }^{\mathrm{A}}$ Saoussen Ftouh, ${ }^{\mathrm{B}}$ Patrick Langford, ${ }^{\mathrm{C}}$ Timothy $\mathrm{S}$ Chesser ${ }^{\mathrm{D}}$ and Antony Johanssen ${ }^{\mathrm{E}}$
}

Hip fracture is a prevalent age-associated occurrence incorporating both medical and surgical need and a major challenge to public health and NHS resources. Effective management requires coordinated collaboration across specialties, professions and services. This concise guideline focuses on interdisciplinary aspects of hip fracture management abstracted from National Institute for Health and Care Excellence (NICE) clinical guideline (CG124), including the concept and implementation of the Hip Fracture Programme, detection and management of comorbidity and delirium, optimal analgesia, timing of surgery, multidisciplinary mobilisation, rehabilitation and hospital discharge. The recently updated National Hip Fracture Database report and NICE quality standard are potential drivers for progress.

KEYWORDS: Comorbidity, hip fracture, interdisciplinary, multidisciplinary, NICE guidance

\section{Introduction}

Hip fracture (a fracture occurring in the area between the edge of the femoral head and $5 \mathrm{~cm}$ below the lesser trochanter) is a major and growing healthcare challenge, linked to population ageing, which requires close collaboration across medical and surgical disciplines.

An annual incidence of about 75,000 (average age 83-84 years) results in health and social care costs of about $£ 2$ billion, and in mortality rates of about $7.1 \%$ at 1 month and $30 \%$ at 1 year, which reflect age-associated comorbidity rather than hip fracture per se. ${ }^{1}$ Prompt, effective analgesia, anaesthesia and surgery, coordinated with structured, medical and multidisciplinary management from presentation to hospital discharge, are therefore needed and have been shown to deliver improved outcomes cost-effectively. Because of the scale of pressure on hospital beds and community services, and the breadth of assessment and management issues, hip fracture is arguably a paradigm of acute hospital care for older people, and of wider relevance for clinical practice and future service provision.

Authors: ${ }^{A}$ emeritus professor of healthcare of the elderly, King's College School of Medicine, London, UK; ${ }^{\text {B }}$ senior research fellow, National Guideline Centre, Royal College of Physicians, London, UK; C technical analyst - surveillance, Centre for Guidelines, National Institute for

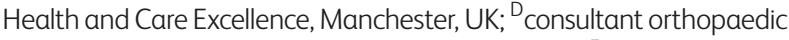
trauma surgeon, North Bristol NHS Trust, Bristol, UK; ${ }^{\text {E }}$ consultant orthogeriatrician, Trauma Unit, University Hospital of Wales, Cardiff, UK

\section{Scope and purpose}

The National Institute for Health and Care Excellence (NICE) guideline Hip fracture: management ${ }^{2}$ (CG124) was published in July 2011, and reviewed in 2013 and 2015. In response to the 2015 review, a partial update limited to surgical management of intracapsular fractures (including the indication for total hip replacement and the management of undisplaced fractures) is scheduled for publication in 2017. ${ }^{3}$ The corresponding quality standard (QS16) was first published in March 2012 ${ }^{4}$ and updated in November 2016. ${ }^{5}$

This concise guideline focuses selectively on recommendations with substantial implications for current and future interdisciplinary ${ }^{\mathrm{a}, 6}$ hospital practice and service delivery. It does not cover detailed guidance on surgical procedures or radiological diagnosis, but places particular emphasis on the updated QS16 priorities for interdisciplinary management (including timing of surgery).

\section{Recommendations}

\section{Hip Fracture Programme}

From admission, offer patients a formal, acute orthogeriatric or orthopaedic ward-based Hip Fracture Programme that includes all of the following:

$>$ orthogeriatric assessment

$>$ rapid optimisation of fitness for surgery

$>$ early identification of individual goals for multidisciplinary rehabilitation to recover mobility and independence, and to facilitate return to pre-fracture residence and long-term wellbeing

$>$ continued, coordinated, orthogeriatric and multidisciplinary review

$>$ liaison or integration with related services, particularly mental health, falls prevention, bone health, primary care and social services

$>$ clinical and service governance responsibility for all stages of the pathway of care and rehabilitation, including those delivered in the community.

The National Institute of Health and Care Excellence (NICE) has accredited the process used by the Royal College of Physicians to produce the concise clinical guidelines published in Clinical Medicine with effect February 2010 to March 2018 (abstracted guidance) and July 2013 to July 2018 (de novo guidance). More information on accreditation can be viewed at: www.nice.org.uk/ about/what-we-do/accreditation. 
The Hip Fracture Programme (HFP) concept is readily misunderstood as merely a generic label for a service. On the contrary, it is a comprehensive evidence-based model of immediate and ongoing interdisciplinary assessment, management and discharge planning. In place of more traditional models of orthopaedic care followed by routine transfer for rehabilitation elsewhere (eg orthopaedic or general rehabilitation units), assessment and care are expedited and organised in the acute hospital, promoting direct and prompt discharge (wherever possible) to the patient's normal home setting. Its cost-effectiveness versus traditional models has been established following the latest CG124 evidence review. ${ }^{7,8}$

The medical and multidisciplinary 'orthogeriatrician' role was developed by physicians in clinical gerontology, but is applicable to the care of hip fracture in adults of all ages.

\section{Minimise risk of delirium}

Deliver care that minimises the patient's risk of delirium and maximises their independence by:

$>$ actively looking for cognitive impairment when patients first present with hip fracture

$>$ reassessing patients to identify delirium that may arise during their admission.

The prevalence of delirium is high with hip fracture, it is under-recognised, may signal related comorbidity and is not confined to those with prior cognitive impairment. ${ }^{9}$ A case finding approach is essential, with immediate recognition of common presentations, appropriate use of a validated tool (eg the 4 AT rapid assessment test) ${ }^{10}$ and management following current NICE guidance. ${ }^{11}$

\section{Identify and treat comorbidities}

Identify and treat correctable comorbidities immediately so that surgery is not delayed by:

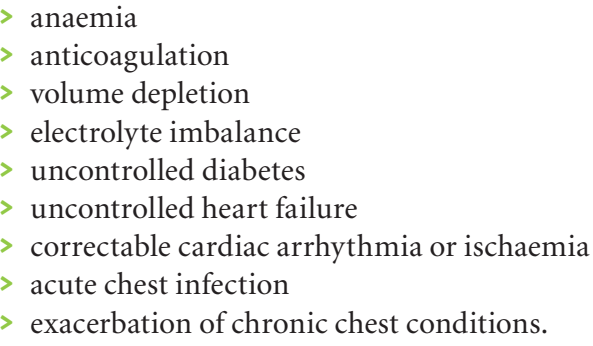

Historically, many of the above have served as unjustified pretexts for surgical delay, but skilled interdisciplinary (especially anaesthesia - medicine) preoperative assessment has now minimised such inappropriate 'contraindications' to prompt surgery.

\section{Provide immediate pain relief}

Offer immediate and continuing analgesia to patients presenting at hospital with suspected hip fracture, including people with cognitive impairment.

Ensure analgesia is sufficient to allow movements necessary for investigations (as indicated by the ability to tolerate passive external rotation of the leg), and for nursing care and rehabilitation.
Offer paracetamol every 6 hours pre- and postoperatively unless contraindicated, with additional opioids pre- and postoperatively if this does not provide sufficient pain relief.

Consider adding nerve blocks (administered by trained personnel) if paracetamol and opioids do not provide sufficient preoperative pain relief, or to limit opioid dosage.

Pain relief is understandably top priority for patients, but is also critical to effective clinical management both preoperatively and throughout rehabilitation. Paracetamol's effectiveness has been historically somewhat underestimated. Excessive opioid use impairs outcomes and is to be avoided, along with non-steroidal anti-inflammatory agents. The availability and use of nerve blocks is increasing, but evidence to support their routine use is still awaited.

\section{Avoid surgical delay}

Perform surgery (Table 1) on the day of, or the day after, admission, on a planned trauma list with consultant or senior staff supervision of trainee and junior members of the anaesthesia, surgical and theatre teams.

Offer patients a choice of spinal or general anaesthesia after discussing the risks and benefits of each.

Operate on patients with the aim to allow them to fully weight bear (without restriction) in the immediate postoperative period.

Improved outcome and the cost-effectiveness of early surgery are well established in the evidence. The cost-effectiveness of surgery before 48 hours is clear, and for any time-tosurgery threshold down to 24 hours, reducing the delay to

\section{Table 1. Surgical procedures - summary of CG124} recommendations for hip fracture ${ }^{3}$

\begin{tabular}{|c|c|}
\hline $\begin{array}{l}\text { Undisplaced intracapsular } \\
\text { fracture }\end{array}$ & $\begin{array}{l}\text { Internal fixation taken as } \\
\text { normative - therefore not covered } \\
\text { in the guideline* }\end{array}$ \\
\hline \multirow[t]{6}{*}{$\begin{array}{l}\text { Displaced intracapsular } \\
\text { fracture }\end{array}$} & $\begin{array}{l}\text { Perform replacement arthroplasty } \\
\text { (hemiarthroplasty or total hip } \\
\text { replacement) }\end{array}$ \\
\hline & $\begin{array}{l}\text { Use cemented implants in } \\
\text { patients undergoing surgery with } \\
\text { arthroplasty }\end{array}$ \\
\hline & $\begin{array}{l}\text { Offer total hip replacements* to } \\
\text { patients who: }\end{array}$ \\
\hline & $\begin{array}{l}\text { were able to walk independently } \\
\text { out of doors with no more than } \\
\text { the use of a stick and }\end{array}$ \\
\hline & $\begin{array}{l}\text { are not cognitively impaired } \\
\text { and }\end{array}$ \\
\hline & $\begin{array}{l}>\text { are medically fit for anaesthesia } \\
\text { and the procedure }\end{array}$ \\
\hline $\begin{array}{l}\text { Trochanteric fractures above } \\
\text { and including the lesser } \\
\text { trochanter (AO classification } \\
\text { types } A 1 \text { and } A 2 \text { ) }\end{array}$ & $\begin{array}{l}\text { Use extramedullary implants, such } \\
\text { as a sliding hip screw in preference } \\
\text { to an intramedullary nail }\end{array}$ \\
\hline Subtrochanteric fracture & Use an intramedullary nail \\
\hline
\end{tabular}


within the threshold has shown benefit. Postponement of surgery carries increased risk of complications, as well as prolongation of pain, and the need for repeated preoperative fasting. The recommendation to operate no later than the day after admission incorporates pragmatic, organisational and humanitarian considerations. Investment in planned trauma lists (with senior supervision) to achieve this becomes demonstrably cost-effective within 2 years of implementation.

No compelling evidence has so far been provided for superior clinical outcomes with either regional (spinal) or general anaesthesia. Patient experience is of central importance; therefore the open offer of choice following explanatory discussion is entirely appropriate.

Surgery that permits immediate postoperative unrestricted full weight bearing is crucial to the safe, positive benefits of early mobilisation.

\section{Early mobilisation}

Offer patients a physiotherapy assessment and, unless medically or surgically contraindicated, mobilisation on the day after surgery and at least once daily thereafter, with regular physiotherapy review.

Measured at day 7, early mobilisation improves walking distance and increases independence in transferring, although robust evidence showing benefit on discharge destination, mortality and cost-effectiveness is yet to be obtained. ${ }^{12}$

\section{Early supported discharge}

Consider early supported discharge as part of the HFP, provided the HFP multidisciplinary team remains involved, and the patient

$>$ is medically stable and

$>$ has the mental ability to participate in continued rehabilitation and

$>$ is able to transfer and mobilise short distances and

$>$ has not yet achieved their full rehabilitation potential, as discussed with patient, carer and family.

Early supported discharge, a defined secondary/primary care partnership approach to early postoperative community-based multidisciplinary rehabilitation for patients fulfilling the above criteria, achieves a greater improvement in daily living activities from the outset, improves measured falls risk at 4 months and, despite longer overall rehabilitation, reduces costs by reducing hospital length of stay. ${ }^{13}$

\section{Intermediate care}

Only consider intermediate care (continued rehabilitation in a community hospital or residential care unit) if all of the following criteria are met:

$>$ intermediate care is included in the HFP and

$>$ the HFP team retains the clinical lead, including patient selection, agreement of length of stay and ongoing objectives for intermediate care and

$>$ the HFP team retains the managerial lead, ensuring that intermediate care is not resourced as a substitute for an effective acute hospital Programme.
The cautionary emphasis of this recommendation reflects:

1 the absence to date of specific evidence for the effectiveness and cost-effectiveness of hip fracture management involving intermediate care beds;

2 the likelihood that premature discharge to social residential care settings may reduce hospital stay at the expense of greater long-term dependency and overall health costs; and

3 the cost-effectiveness of the HFP model compared with routine transfer to other units for rehabilitation.?

\section{Limitations of the guideline}

For some recommendations where the strength of supporting evidence is weaker than for others, they are based in part on the experience and opinion of the Guideline Committee. Some corresponding research recommendations include the clinical and cost effectiveness of:

> regional versus general anaesthesia on postoperative morbidity

$>$ additional intensive physiotherapy and/or occupational therapy (for example progressive resistance training)

$>$ early supported discharge on mortality, quality of life and functional status in patients admitted from a care home.

\section{Implications for implementation}

CG124 constitutes the core guidance underpinning a national audit - the National Hip Fracture Database (NHFD). This clinically led, web-based audit of hip fracture care and secondary prevention includes nearly $95 \%$ of all cases presenting to all 177 acute trauma units in England, Wales and Northern Ireland, and allows the process and outcome of care to be monitored and improved. ${ }^{1}$ Engagement with the NHFD is assumed in QS16, and has been a major impetus in driving up standards since its inception in 2007.

The latest 2015/16 NHFD report ${ }^{1}$ documented several such improvements but also highlighted fundamental deficiencies, for example:

$>$ Traditional models of care, rather than the HFP, continued to compromise performance in seven units, and the HFPs in many other units appeared focused disproportionately on acute care. Many units claim to have an HFP, but it is not clear that patients actually receive all of the specified elements. A composite measure of best clinical practice is now in place as an outcome indicator for the NHS Outcomes Framework $^{14}$ to address this.

> There remains unacceptable variation in achieving the recommended time to surgery, with different units reporting figures ranging from $16.7 \%$ to $92.8 \%$.

$>$ Rates of spinal or general anaesthesia vary immensely between hospitals (ranging from $92.0 \%$ of patients receiving spinal in some units to $92.0 \%$ receiving general in others). This may suggest that choice of anaesthesia continues to be driven by the preferences of individual anaesthetists rather than the recommended informed patient choice.

> HFP teams still have a very limited role in monitoring or influencing their patients' post-discharge care, with only $5.6 \%$ having community team representation at clinical governance meetings. 
Given the scale of health and health-economic implications of hip fracture for patients and for the wider healthcare system, further progress is clearly still required. Continued NHFD audit, reinforced by QS16, constitutes an ongoing impetus of growing international recognition. Cost-effective hip fracture management presents a model of wider successful care provision for an ageing population.

\section{Conflicts of interest}

$\mathrm{AJ}$ is clinical lead for the NHFD, and CS is a member of its Advisory Committee. TC is a board member of the FFFAP. PL was the technical analyst for the 2015 guideline review, but was not involved with the 2016 quality standard update.

\section{Acknowledgements}

The contribution of all Guideline Development Group members to the development and review of CG124 is gratefully acknowledged.

\section{Note}

a 'Interdisciplinary' is used throughout to encompass the organised collaborative synthesis of all disciplines (including medicine, surgery, anaesthesia and rehabilitation) within a common approach to hip fracture management. 'Multidisciplinary' refers specifically to the regular organised team approach to assessment and rehabilitation. ${ }^{6}$

\section{References}

1 Royal College of Physicians. National Hip Fracture Database annual report 2016. London: RCP, 2016.

2 National Institute for Health and Care Excellence. Hip fracture: management. NICE clinical guideline No 124. London: NICE, 2011.

3 National Institute for Health and Care Excellence. Surveillance report-Hip fracture. NICE guideline No 124. London: NICE, 2015.

4 National Institute for Health and Care Excellence. Hip fracture in adults. NICE quality standard No 16. Manchester: NICE, 2012.
5 National Institute for Health and Care Excellence. Hip fracture in adults. NICE quality standard No 16. Manchester: NICE, 2016.

6 Choi BCI, Pak AW. Multidisciplinarity, interdisciplinarity and transdisciplinarity in health research, services, education and policy: 1. Definitions, objectives, and evidence of effectiveness. Clin Invest Med 2006;29:351-64.

7 National Institute for Health and Care Excellence. 4-year surveillance 2015 - Hip fracture. NICE guideline No 124. Appendix A: decision matrix. Available online at www.nice.org.uk/guidance/cg124/evidence/ surveillance-review-decision-matrix-december-2015-2190593774 [Accessed 1 November 2016].

8 Grigoryan KV, Javedan H, and Rudolph JL. Orthogeriatric care models and outcomes in hip fracture patients: a systematic review and meta-analysis. J Orthop Trauma 2014;28:e49-55.

9 Marcantonio ER, Flacker JM, Wright RJ, Resnick MN. Reducing delirium after hip fracture, a randomized trial. J Am Geriatr Soc 2001;49: 516-22.

10 MacLullich A, Ryan T, Cash H. The 4AT. Available online at www. the4at.com/ [Accessed 1 November 2016].

11 National Institute for Health and Care Excellence. Delirium: prevention, diagnosis and management. NICE clinical guideline No 103. Manchester: NICE, 2010.

12 Oldmeadow LB, Edwards ER, Kimmel LA et al. No rest for the wounded: Early ambulation after hip surgery accelerates recovery. ANZ J Surg 2006;76:607-11.

13 Crotty M, Whitehead CH, Gray S, Finucane PM. Early discharge and home rehabilitation after hip fracture achieves functional improvements: a randomised controlled trial. Clin Rehabil 2002;16:406-13.

14 Department of Health. NHS Outcomes Framework: at-a-glance. List of outcomes and indicators in the NHS Outcomes Framework for 2016-17. London: Department of Health, 2016.

Address for correspondence: Professor C G Swift, Clinical Age Research Unit, King's College Hospital, Denmark Hill, London SE5 9RS, UK.

Email: cameron.swift@kcl.ac.uk 